\title{
Appareil
}

$21 \mid 2019$

Le théâtre comme appareil

\section{Quel appareil théâtral ?}

1. L'appareil scénique

Stéphane Poliakov

\section{(2) OpenEdition}

Journals

Édition électronique

URL : http://journals.openedition.org/appareil/3170

DOI : 10.4000/appareil.3170

ISSN : 2101-0714

Éditeur

MSH Paris Nord

Référence électronique

Stéphane Poliakov, «Quel appareil théâtral ? », Appareil [En ligne], 21 | 2019, mis en ligne le 11 juillet 2019, consulté le 30 juillet 2020. URL : http://journals.openedition.org/appareil/3170 ; DOI : https:// doi.org/10.4000/appareil.3170

Ce document a été généré automatiquement le 30 juillet 2020.

\section{(9) $\odot \Theta \Theta$}

Appareil est mis à disposition selon les termes de la Licence Creative Commons Attribution - Pas d'Utilisation Commerciale - Pas de Modification 4.0 International. 


\title{
Quel appareil théâtral ?
}

\author{
1. L'appareil scénique
}

\section{Stéphane Poliakov}

« Dût tout cet appareil retomber sur ma tête

Il faut parler. "

Racine, Iphigénie en Aulide, acte III, scène V.

1 Jean-Louis Déotte a voué son œuvre à la théorisation de la notion d'appareil, réalisant par-là la définition deleuzienne de la philosophie comme « création de concepts ». C'est une entreprise commune que d'en montrer la fertilité, le sens et parfois les limites. Cela permet aussi de mettre à l'épreuve l'extension de ce concept esthétique et la manière dont cette idéalité même - un concept esthétique - est possible malgré la restriction kantienne et au-delà de la possibilité formaliste soutenue par Wölfflin dans les Principes fondamentaux de l'histoire de l'art. Nous souhaitons faire jouer littéralement la notion d'appareil dans le champ théâtral. D'une part, parce que le théâtre est un art avec sa singularité temporelle et spatiale, son caractère collectif ; d'autre part, parce que le mot même d'appareil est en partie théâtral. Nous commencerons notre recherche par la langue et la culture théâtrale françaises (le terme a une origine latine et italienne) avant d'aborder, dans un deuxième volet, le champ théâtral russe. "Théâtre " n'aura pas ici de portée essentielle, mais une extension factuelle - ce qui est nommé théâtre. Il a une dimension matérielle, historique, un contenu, sinon une forme. Nous ne travaillons pas à une définition du théâtre. Aussi bien en français que dans la langue russe, théâtre (teatr) est immédiatement évocateur et vivant, englobant l'acteur, la mise en scène, le spectacle, l'œuvre dramatique, la scénographie, l'entreprise théâtrale et même l'édifice selon une polysémie générique du terme dans les deux langues (en russe, il désigne aussi la troupe, la compagnie). Appareil est plus restrictif. Je propose donc tout d'abord d'explorer le sens français d'appareil scénique qui fait signe vers la scénographie et la matérialité inorganique de la scène. 


\section{L'appareil scénique : origine lexicale}

2 «Appareil » évoque l'apprêt, la chose ornée, parée, ordonnée. Le terme est susceptible de degrés jusqu'au plus " simple appareil ${ }^{1}$ ", c'est-à-dire sa quasi-absence, qui serait au théâtre la nudité de la scène ou du corps disponible de l'acteur. C'est alors une préparation minimale, comme au pied levé, là où la technique rejoint presque la nature. Furetière définit le terme entre apprêt et apparat (mais aussi, en un réseau plus lointain et non moins théâtral, parade), qui sont des termes apparentés :

APPAREIL, s. m. Ce qu'on prepare pour faire une chose plus ou moins solemnelle. L'entrée du Roy aprés son mariage s'est faite avec beaucoup d'appareil \& de magnificence : on travaille à l'équipage, à l'appareil des ceremonies de son Sacre. je croyois qu'il y auroit aujourd'huy un festin en une telle maison, mais je n'y vois aucun appareil. On dit aussi, un grand appareil de guerre. Ce mot vient du verbe apparare $^{2}$.

Appareil a dans la langue française, depuis le XII siècle («apareiz», «apareilz», « appareaul $\left.x^{3} »\right)$, le sens d'une configuration technique et instrumentale disposée pour un usage précis, en particulier une cérémonie. Au théâtre, c'est une machine ou un élément de machinerie, mais aussi l'ensemble des décors, la décoration, les costumes, les accessoires, les masques, etc., tout ce qui fait le faste de la scène. La Salle des Machines du Château des Tuileries est conçue au XvII e siècle pour les apparitions, les "vols ${ }^{4}$ », les coups de tonnerre et les ruissellements d'eau, les effets de nuages et d'orages, les jeux pyrotechniques et les changements à vue et successifs au moyen de mécanismes simples - roues, poulies, tissus, maniement de poids et contrepoids, appareillage de fer. C'est le monde des ingénieurs de théâtre traditionnellement rapproché de la navigation - la langue signale l'usage du terme dans ce contexte («Appareil de mâts \& de voiles ${ }^{5}$ »). Il est sans doute né dans l'Italie du $\mathrm{XvI}^{\mathrm{e}}$ siècle de Léonard de Vinci à Bernardo Buontalenti ${ }^{6}$ en passant par Sebastiano Serlio, et c'est d'Italie qu'il est passé en France par la volonté politique de François $\mathrm{I}^{\mathrm{er}}$, Richelieu, puis Mazarin et Louis XIV. Ainsi, le « Trattato sopra le scene » de Serlio, inclus dans son Second livre de perspective, paraît en langue italienne et française à Paris en 1545. C'est le premier écrit moderne de scénographie. Dès ses premières lignes, il parle de la découverte merveilleuse pour l'œil et l'esprit de «lo apparato di una scena » ce que l'édition bilingue traduit de la façon suivante :

Entre les choses faites par la main des hommes dont l'on se peut émerveiller et recevoir contentement d'œil, avec satisfaction de pensée, à mon jugement, c'est l'appareil d'une scène quand on vient à le découvrir. La raison est, que l'on y voit en peu d'espace aucuns palais, dressés par art de perspective, avec grands temples et divers maisonnages proches et lointains de la vue, places belles et spacieuses, décorées de plusieurs édifices, rues longues et droites, croisées de voies traversantes, arcs de triomphe, colonnes hautes à merveilles, pyramides, obélisques et mille autres singularités, enrichies de lumières grandes, moyennes et petites, ordonnées par tel artifice qu'elles semblent autant de pierres précieuses rendant une lumière admirable, comme seraient rubis, diamants, saphirs, émeraudes et choses semblables ${ }^{7}$.

4 Sa distinction de trois "maniere » dans les "apparati delle comedie ${ }^{8}$ " (scènes comique, tragique et satirique) ainsi que les gravures qui les illustrent sont fondamentales dans l'histoire du théâtre occidental (fig. 1). 
Fig. 1 Sebastiano Serlio, «Scena Comica »

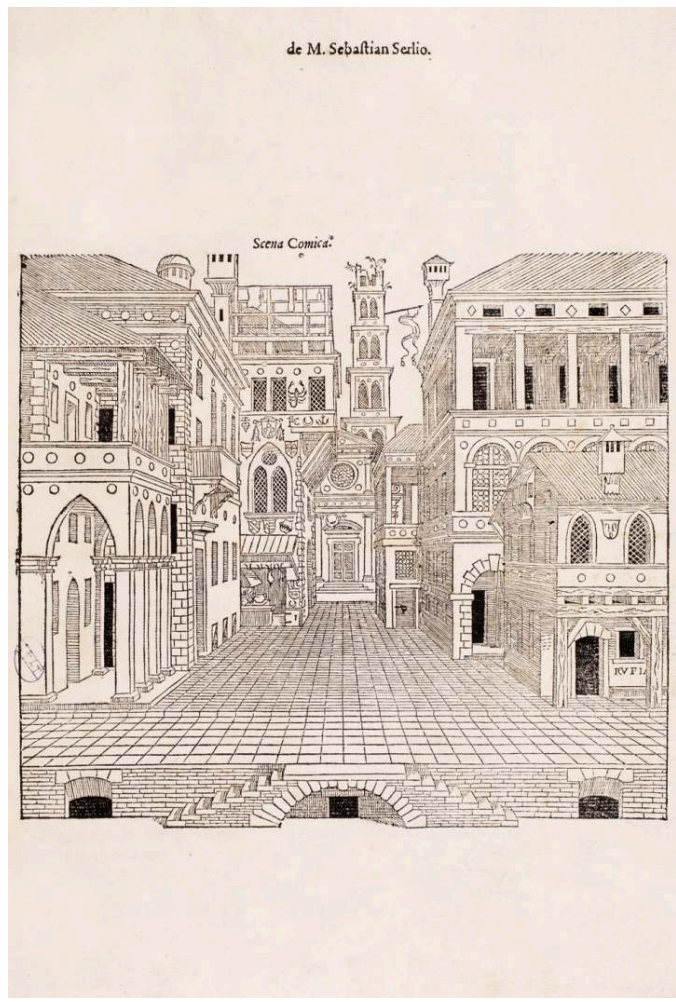

Gravure sur bois in Second Livre de Perspective, Paris 1545 ; détail Source. [dr]

5 La représentation, en 1641, de la tragi-comédie Mirame de Desmarets de Saint-Sorlin, avec des vers de Richelieu lui-même, au Théâtre du Palais-Royal est un autre jalon de ce passage. Elle est documentée par des gravures de Stefano della Bella et une célèbre estampe attribuée au peintre Jean de Saint-Igny (fig. 2). 
Fig. 2 Jean de Saint-Igny, Représentation de Mirame au Palais-Cardinal devant Louis XIII, Anne d'Autriche et Richelieu, le 24 janvier 1641, estampe

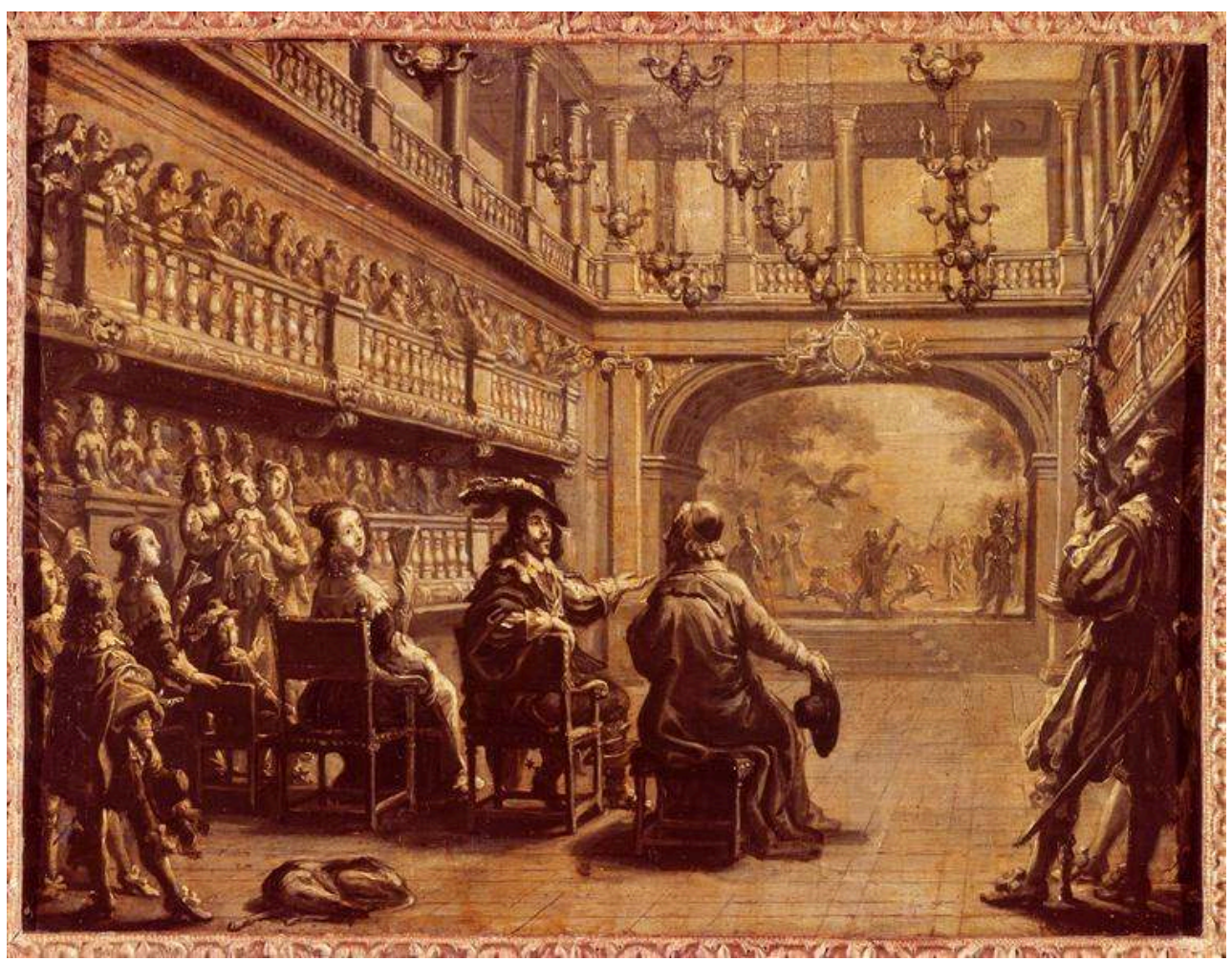

Source. Paris, Musée des arts décoratifs/Photo. RMN-Grand Palais/Agence Bulloz

Suivent La Finta pazza au Petit-Bourbon en 1645, Andromède de Corneille en 1650, Psyché de Molière et Corneille (Thomas et Pierre) en 1671, ces deux derniers spectacles ayant été conçus pour la Salle des Machines des Tuileries. Les ingénieurs-scénographes de ces spectacles sont Giacomo Torelli, Carlo Vigarani, plus tard Jean Bérain. Le lien lexical avec l'Italie est direct. En 1644 sont publiés les Apparati scenici per lo teatro novissimo di Venetia, dédiés au Cardinal Barberini, qui figurent les décors de Torelli pour l'opéra la Venere Gelosa. Nous sommes aux prémices du théâtre baroque commercial qui s'invente à Venise et s'exporte à Paris par la «magie » de Torelli. Ce dernier se relie à la tradition française par la création des décors à machines d'Andromède ${ }^{9}$. C'est l'origine du terme appareil scénique dans la langue française $\mathrm{du} \mathrm{XVII}^{\mathrm{e}}$ au XIX ${ }^{\mathrm{e}}$ siècle.

7 Cet usage est attesté chez Voltaire, artisan du grand spectacle de cour avec La Princesse de Navarre, fruit de sa collaboration avec Rameau en $1745^{10}$ :

$\mathrm{Au}$ reste, quand je parle d'une action théâtrale, je parle d'un appareil, d'une cérémonie, d'une assemblée, d'un événement nécessaire à la Pièce, \& non pas de ces vains spectacles plus puériles que pompeux, de ces ressources du décorateur qui suppléent à la stérilité du Poëte, \& qui amusent les yeux, quand on ne sait pas parler aux oreilles \& à l'âme ${ }^{11}$.

On voit ici se dessiner une dichotomie intéressante entre l'appareil décoratif extérieur (vain et pompeux, purement visuel) et l'accent rhétorique sur l'agencement nécessaire et fécond de la composition. Elle s'insère à son tour dans la tradition aristotélicienne, si prégnante en France et en Italie, d'une opposition entre l'opsis (le pur spectacle) et l'efficacité pathique du «système des faits » (sustasis tôn pragmatôn $)^{12}$. 


\section{À travers l'Encyclopédie, vers un refus de « l'appareil de la représentation »}

9 L'Encyclopédie permet de cartographier la notion en son sens classique (plus de six cents occurrences du terme) et d'en mesurer les applications au théâtre. Le sens chirurgical est de loin le plus fréquent: on prépare, on applique ou l'on lève un appareil. Puis, vient le sens chimique par lequel on approche le sens moderne puisqu'il est question d'appareil de distillation, de désigner un fourneau ou même un thermomètre (voir ce terme). On mettra de côté le sens lié au lexique de la chasse (piège, apprêt), de la cuisine, de la philologie (appareil critique) et même le sens esthétique en architecture qui se retrouve dans les articles "Pierre» et "Maçonnerie", mais dont l'usage n'est pas le plus fréquent.

10 Plus intéressant pour notre champ est le lien entre appareil et triomphe dans de nombreux articles sur l'histoire ancienne rédigés par Jaucourt ${ }^{13}$. Le terme apparait en référence aux Entrées royales, au faste, à "l'appareil de la royauté », aux instruments du sacre. Dans le contexte des Lumières, "appareil » a un sens ambivalent. Il renvoie à l'éclat, une sorte de perfection extérieure, acceptée en bonne part, pour les merveilles de la nature lorsqu'il s'agit de l'appareil corporel, des muscles ou de l'assemblage des organes du hérisson, de l'hippopotame, etc. Mais s'il manifeste la grandeur du pouvoir, il vise surtout à effrayer le peuple. «Appareil» prend ainsi des couleurs négatives : c'est le monde de l'apparence, tantôt grandiose, dans les fêtes de cour ou la splendeur du culte, tantôt effrayante quand il s'agit de l'appareil du tribunal ou du supplice ${ }^{14}$. L'appareil est souvent "vain». Diderot parle de "l'appareil de la religion» ou de " l'appareil effrayant des miracles et des prodiges". L'appareil est "lugubre», renvoie à la superstition, la torture et figure dans l'article «Inquisition». Comme le luxe, il est " étalé », écrit Voltaire par deux fois dans l'article "Faste ». L'article "Fêtes à la cour de France » est une description précise du sens théatral et politique qui rejoint celui de « pompe » car les deux termes se définissent mutuellement :

POMPE, s.f. (Gramm.) appareil somptueux, employé pour rendre quelqu'action publique plus solemnelle \& plus recommandable. C'est l'art d'en imposer aux yeux. Une pompe funèbre, c'est l'appareil de l'inhumation d'un grand; sa vanité, pour ainsi dire, lui survit encore ${ }^{15}$. [...]

C'est ainsi que s'établit la définition la plus générique :

APPAREIL, s. m. signifie proprement une préparation formelle à quelque acte public \& solennel. Voyez PREPARATION.

Nous disons l'appareil d'une fête ou d'un couronnement; qu'un Prince a fait son entrée avec beaucoup d'appareil \& de magnificence ${ }^{16}$.

11 À Genève, selon d'Alembert, les choses se font avec "beaucoup d'appareil » dans l'exercice de la justice ou "sans apparei ${ }^{17}$ » lorsqu'il s'agit des funérailles. On se souvient que c'est à cet article que Rousseau répond en 1758 dans sa célèbre Lettre sur les spectacles.

Peut-être est-ce encore Voltaire qui résume le mieux le sens théâtral, moral et politique en écrivant dans l'article « Gloire » :

On a vû des souverains qui ayant une gloire réelle, ont encore aimé la vaine gloire, en recherchant trop les loüanges, en aimant trop l'appareil de la représentation ${ }^{18}$.

Entre accessoire et arrangement ordonné, voire système, la notion est au carrefour de ce qui est parfaitement nommé " appareil de la représentation ». Entre excès et défaut, c'est un mal nécessaire, lié au pouvoir et au théâtre de la cour. Le sens progressiste et négatif 
est bien exprimé par Jaucourt à l'article « Méthode» à propos du fatras rhétorique : "On donne dans un appareil inutile, plus propre à brouiller les idées qu'à y répandre de la lumière ${ }^{19}$. » L'appareil « auguste » ou « fastueux » devient ainsi « ridicule », «faux » ou « horrible » pour reprendre des syntagmes attestés. Si l'appareil désigne les insignes ou les oripeaux de la royauté, voire sa face terrible et menaçante, c'est qu'il ne recouvre plus dans l'Encyclopédie le sens de vertu et de justice. Sans doute même s'y oppose-t-il. En définitive, le terme recoupe assez bien le sens de vanité et de facticité un peu grandiloquente qu'a encore dans notre langue l'adjectif "théatral ». L'appareil se dissout lorsqu'il perd sa valeur constructive de présentation pour devenir ornemental au risque d'être superflu. Au point de vue politique et esthétique, il est récusé dans son association constante au théâtre de la cour.

On voit donc dans la langue le juste reflet des mouvements qui affectent la vie théâtrale et celle des spectacles ainsi que la sphère publique. Dans la préface de Sémiramis, l' appareil de la scène est jugé indispensable par Voltaire qui contribue, par sa réaction, après l'échec de la première représentation de sa pièce, à débarrasser la scène du Théâtre-Français des spectateurs privilégiés pour l'ouvrir à une visualité et une ampleur nouvelle, depuis la salle. Il y parvient, à partir de $1759^{20}$, par l'intervention du grand acteur Lekain, interprète de ses tragédies aux côtés de Mlle Clairon. Bien vite, dans le sillage des théories de Diderot, de Noverre, de Beaumarchais sur la pantomime, le tableau, le drame bourgeois, le genre sérieux, c'est la vie plus que la pompe qui devient l'idéal du théâtre. En 1767, Beaumarchais écrit ainsi dans son Essai sur le genre dramatique sérieux:

Pour que le genre sérieux ait toute la vérité qu'on a droit d'exiger de lui, le premier objet de l'auteur doit être de me transporter si loin des coulisses, et de faire si bien disparaître à mes yeux tout le badinage d'acteurs, l'appareil théâtral, que le souvenir ne puisse pas m'atteindre une seule fois dans tout le cours de son drame ${ }^{21}$.

Dans le sillage de Diderot, il s'agit de préférer la prose aux vers, le salon au «palais à volonté ", son propre temps à la Grèce ou à Rome, et l'homme simple et ordinaire au roi. Refuser l'appareil est un parti-pris contre « les sentences et les plumes du tragique ${ }^{22}$ ».

\section{Un paradigme philosophique et théâtral}

Il serait vain, cependant, de limiter la notion d'appareil au théâtre à son usage classique entre 1640 et 1760. Dans le sillage de J.-L. Déotte, nous cherchons à établir un réseau de sens. Il faut donc élargir les frontières temporelles et la définition de l'appareil scénique. Il faut lui adjoindre "machine", qui en manifeste la part technique, mais aussi " décoration ", «tableau » et même "théâtre » en tant qu'il désigne l'espace scénique. $\mathrm{Au} \mathrm{XIX}^{\mathrm{e}}$ siècle, Arthur Pougin, dans son dictionnaire théâtral, lui donne une place pleine d'ambiguïté. L'appareil est à la fois «matériel » et « immatériel », entre technique - «l' illusion» nécessaire - et réalisme (la "réalité des choses»), dans une tension constitutive avec la "personnalité humaine», terme qui voile efficacement celui d'acteur. Si la définition est matérielle, elle se qualifie, dans le même temps, comme impression insaisissable :

Appareil scénique. - On doit entendre par «appareil scénique " la réunion de tous les éléments matériels qui concourent à la beauté du spectacle, et dont l'emploi intelligent tend à frapper l'imagination du spectateur et à lui donner le sentiment de la réalité des choses qui sont offertes à sa vue. Décors, costumes, accessoires, 
éclairage, trucs ingénieux, tout, jusqu'au groupement harmonique des masses et à l'habileté de leur mise en mouvement, tout a son rôle dans l'illusion théâtrale, et, à part la personnalité humaine prise en elle-même et isolément, tout cela fait partie de cette chose insaisissable matériellement, mais dont le sens est très compréhensible qu'on caractérise sous le nom d'appareil scénique ${ }^{23}$.

L'appareil scénique est ici soumis à un principe d'unité et d'ordonnancement technique hérité de l'art de l'ingénieur, mais cette architectonique ou cette belle hiérarchie élude les aléas de l'instabilité humaine. Dans la langue actuelle du théâtre, on utilise plus volontiers le terme de "dispositif ", pour parler des choix scénographiques. Le terme traduit assez bien le russe ustanovka, utilisé par Meyerhold pour sa mise en scène constructiviste du Cocu magnifique de Crommelynck en 1922. "Dispositif» remplace "décors » et complète le terme de "scénographie » qui, selon Aristote, remonte à Sophocle ${ }^{24}$. On a bien là un ensemble de signifiés théâtraux scéniques. L'appareil est un réseau matériel et social. Il est infrastructure et superstructure. Pour le dire avec Walter Benjamin, c'est tout autant une configuration matérielle et sociale qu'une fantasmagorie ${ }^{25}$.

Philosophiquement et esthétiquement, le plus évident est d'arrimer l'appareil scénique à la perspective ${ }^{26}$. L'appareil est un rouage (machinerie), mais aussi la pièce d'une plus grande unité. Les 87 planches du supplément de l'Encyclopédie sur les « Théâtres » et les "Machines de théâtres », qui en dévoilent le fonctionnement (fig.3), participent pleinement de l'appareil perspectif et constructif.

Fig. 3 Machines de Théâtres

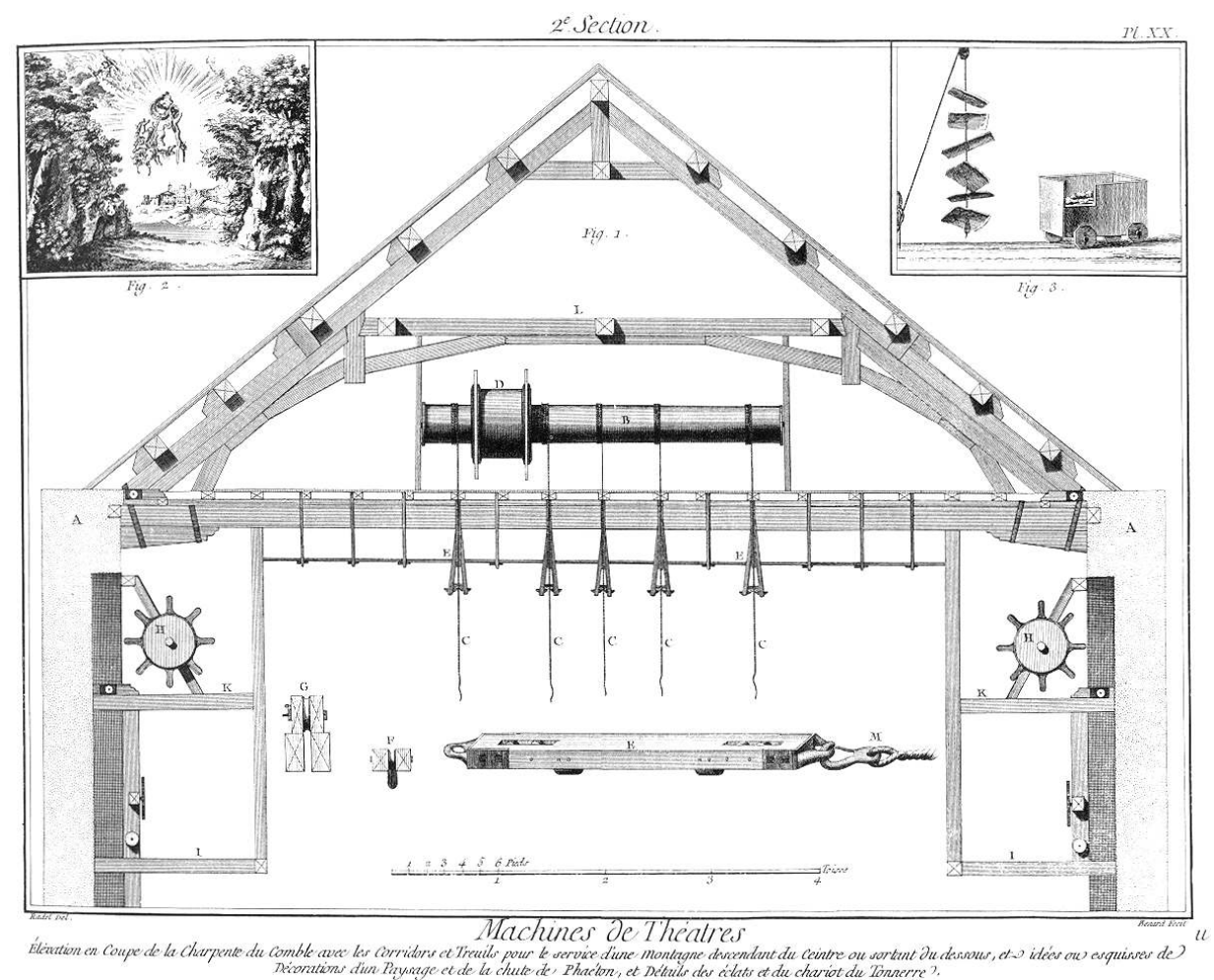

Source. L'Encyclopédie, recueil de planches sur les sciences, les arts libéraux et les arts mécaniques. Théâtres Machines de Théâtre, Planche XX, Paris, 1772. Consultable sur https://gallica.bnf.fr/ark:/ 12148/bpt6k9969f 
C'est l'optique picturale appliquée à l'espace scénique (et à la salle) en fonction d'un point de vue. Serlio, Inigo Jones, Nicola Sabbattini, admiré et édité par Louis Jouvet en 1942, mettent en place un appareillage du théâtre qui mêle peinture, architecture, organisation de la fête princière (puis commerciale). La perspective, instrument optique, devenu scénique, est une machine à voir et à imaginer (les effets passent du merveilleux au grossier en quelques années, selon que «l'illusion » opère ou non). Elle marche avec l'appareil du pouvoir, la cour assemblée, plus tard, la séance théâtrale où l'on est voyant et vu. La perspectiva artificialis est expérimentée dans l'espace (très) réduit, souvent provisoire, de la scène picturale avant de s'ériger en places, façades, alignements, portiques, jardins. Le théâtre ici est un microcosme technique et architectural, fait de peinture et châssis de bois, avec parfois des éléments de carton ou papier mâché, qui se combine au monde du changement par les machines. La perspective unitaire et illusionniste s'allie étrangement à la «musique des yeux ${ }^{27}$ » des changements à vue, quasi instantanés, permis par la machinerie. Cette perception et cette intellection du monde sont admirablement rendues au "Premier soir» de L'Entretien sur la pluralité des mondes par Fontenelle. L'opéra - l'appareillage scénique est une analogie de l'univers, son envers technique, véritable appareil physique et mécanique :

Sur cela, je me figure toujours que la nature est un grand spectacle, qui ressemble à celui de l'opéra. Du lieu où vous êtes à l'opéra, vous ne voyez pas le théâtre tout à fait comme il est: on a disposé les décorations et les machines pour faire de loin un effet agréable, et on cache à votre vue ces roues et ces contrepoids qui font tous les mouvements. Aussi ne vous embarrassez-vous guère de deviner comment tout cela se joue. Il n'y a peut-être que quelque machiniste caché dans le parterre, qui s'inquiète d'un vol qui lui aura paru extraordinaire, et qui veut absolument démêler comme ce vol a été exécuté. Vous voyez bien que ce machiniste-là est assez fait comme les philosophes ${ }^{28}$.

La marquise a bien raison de faire remarquer qu' « à ce compte [...], la philosophie est devenue bien mécanique. » Le mécanisme et bientôt le matérialisme (fût-il « enchanté » chez Diderot) vont tôt faire d'appareiller la perspective à un corps, une conscience, une raison, un jugement; la "vérité » prenant peu à peu la place de "l'illusion » comme catégorie esthétique dominante (de même la vie et le roman se combinent au drame dans les théories de Diderot et Beaumarchais). Diderot semble aller dans le même sens dans son analyse morale. Cette fois, il ne s'agit pas de deviner l'envers du décor, mais de trouver la bonne distance par rapport à la scène depuis la salle :

Dans la grande comédie, la comédie du monde, celle à laquelle j'en reviens toujours, toutes les âmes chaudes occupent le théâtre; tous les hommes de génie sont au parterre. Les premiers s'appellent des fous; les seconds, qui s'occupent à copier leurs folies, s'appellent des sages ${ }^{29}$.

21 Paradoxalement, l'art de l'acteur renverse chez Diderot ce paradigme distancié de la scène du monde. Vues depuis la scène, illusion et raison s'équilibrent autrement. L'illusion est dans la salle alors que l'artisan (l'artiste, à cette seule condition d'insensibilité) de la scène - autrement dit le comédien - demeure maître de lui-même. Le prix de l'art sensible dans la salle, c'est le froid calcul du comédien sur la scène qui fait de la déclamation un art et non une simple inspiration ou un mouvement de la nature. À l'appareil de cour succède l'appareil des plaisirs du spectacle et du calcul de ses effets, des sensations, dont l'origine - l'araignée tissant sa toile ou «l'origine du faisceau ", un quasi-Dieu matériel, dans le Rêve de d'Alembert - est le comédien insensible : 
Les hommes chauds, violents, sensibles, sont en scène ; ils donnent le spectacle, mais ils n'en jouissent pas. C'est d'après eux que l'homme de génie fait sa copie. Les grands poètes, les grands acteurs, et peut-être en général tous les grands imitateurs de la nature, quels qu'ils soient, doués d'une belle imagination, d'un grand jugement, d'un tact fin, d'un goût très-sûr, sont les êtres les moins sensibles. [...] Je les vois sans cesse le portefeuille sur les genoux et le crayon à la main. Nous sentons, nous ; eux, ils observent, étudient et peignent. Le dirai-je ? Pourquoi non? La sensibilité n'est guère la qualité d'un grand génie. [...] Ce n'est pas son cœur, c'est sa tête qui fait tout. À la moindre circonstance inopinée, l'homme sensible la perd; il ne sera ni un grand roi, ni un grand ministre, ni un grand capitaine, ni un grand avocat, ni un grand médecin. Remplissez la salle du spectacle de ces pleureurs-là, mais ne m'en placez aucun sur la scène ${ }^{30}$.

Le vieux paradigme de l'imitation permet ainsi de reconstruire un appareil théâtral dans le rapport scène-salle, de maximiser les plaisirs tout en faisant du jeu du comédien un grand art. La perspective peut se construire depuis la scène non seulement visuellement, mais dans l'imagination de l'acteur. Perspective, décors, machines et appareil débordent résolument la scène tout en gardant une part de théâtralité. La scène diderotienne, où évolue le grand acteur, est déjà le produit d'une imitation. Elle est copie des grandes passions et cette médiété la légitime en tant qu'art.

\section{Topologie de la galerie, origine théâtrale des passages}

Le lieu théâtral classique se décompose en "théâtre » proprement dit (pour nous, la scène), parterre (où l'on est debout), amphithéâtre, galeries, loges, paradis, mais aussi coulisses et hors-scène. À partir du dernier tiers du XvIII ${ }^{e}$ siècle, l'espace théâtral devient pleinement citadin : bâtir un théâtre est un geste architectural qui signifie planifier un quartier (les Célestins à Lyon, le théâtre Graslin à Nantes ${ }^{31}$, le théâtre de Besançon, construit par Ledoux, le Grand Théâtre de Bordeaux par Victor Louis, le nouveau Théâtre-Français - actuel Théâtre de l'Odéon - construit par Peyre et De Wailly), des perspectives urbaines, l'aménagement des circulations (notamment des carrosses, fiacres et voitures), de portiques, d'escaliers, de foyers, de galeries. Prenons l'exemple de ce dernier terme.

La "galerie » au théâtre est forme extérieure et intérieure, l'un des éléments de son appareil, notamment commercial. Elle désigne un prix des places, une disposition particulière: d'abord le long des murs longitudinaux des salles perpendiculaires des jeux de paume ou qui en dérivent, puis en ovale, en ellipse, circulaire, divisées (ou non) en loges. La galerie se signale aussi sur scène (La Galerie du Palais de Corneille). Elle se relie au portique et au péristyle qui en sont l'origine architecturale antique et classique (agencement des colonnes) et à son invention architecturale en France et en Italie à la Renaissance $^{32}$. Elle indique un mode de circulation dans le théâtre et ses foyers, mais aussi dans ses alentours. C'est dans ce contexte théâtral qu'apparaissent les premières galeries marchandes urbanisées aux abords des théâtres ou des quartiers de spectacles. C'est au Palais-Royal que naissent les premiers embryons de ces formes analysées par W. Benjamin que sont les "passages" parisiens: Galerie d'Orléans, mais aussi bien d'autres. Après le Passage du Caire et celui des Panoramas, leur développement se fait prioritairement dans le quartier des théâtres et notamment aux abords de l'Opéra de la rue Le Peletier. En témoigne, le fonctionnement, partiellement visible aujourd'hui, de la rue des Colonnes à Paris qui s'articulait à la sortie du Théâtre Feydeau (fig. 4) ou celui 
des passages ou du Passage de l'Opéra qui est l'un des déclencheurs par Aragon et Breton du projet de Benjamin ${ }^{33}$.

Fig. 4. Le Théâtre Feydeau

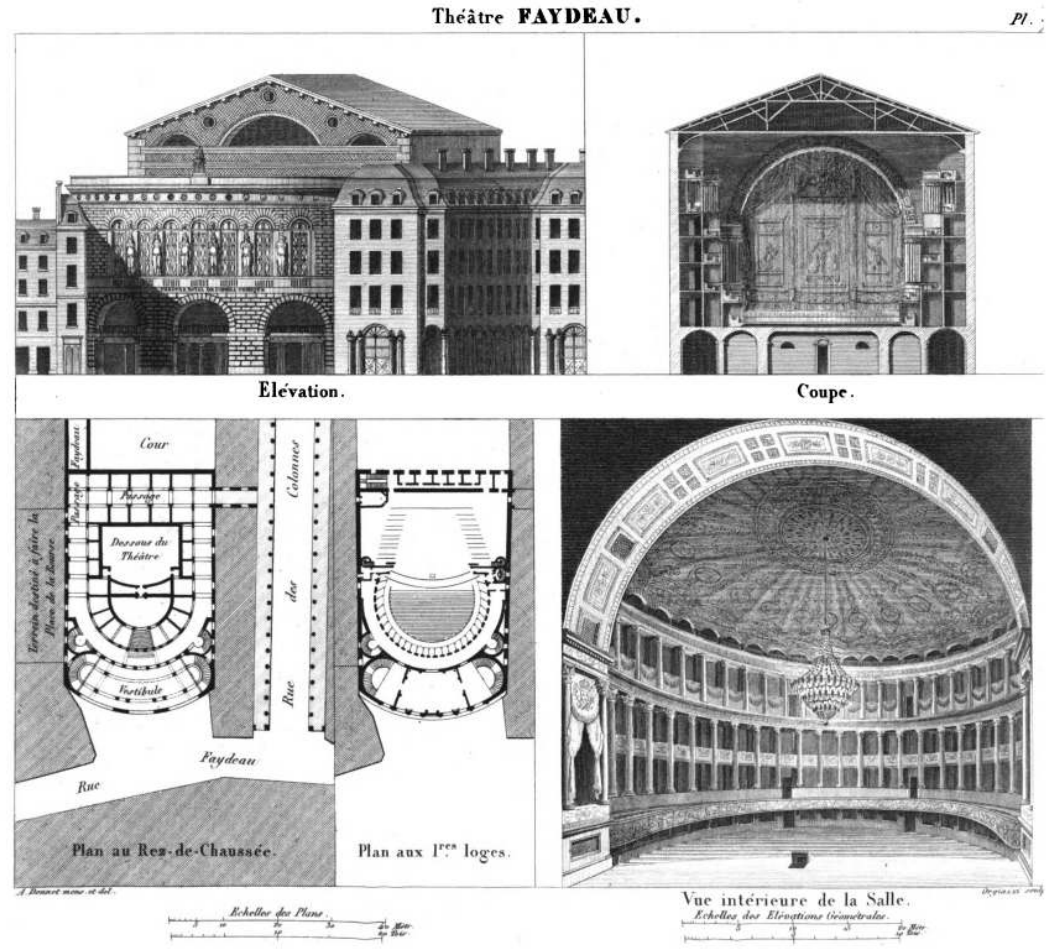

Élévation, Coupe et élévation, Planimétrie du théâtre dans le parcellaire urbain avec la rue des Colonnes, vue intérieure de la salle (axonométrie) in Alexis Donnet (dessins) et J. Orgiazzi (gravure), Architectonographie des théâtres de Paris..., Paris, Didot l'ainé, 1821.

Source. View at Google Books. Ghent University Library, plate 7.0CLC 628572489.

ernementation révèle un fonctionnement du parcellaire urbain, une utopie (Fourrier), une réalité du commerce (la boutique), un mode de circulation (la flânerie). C'est par-là que l'on peut sentir les liens du monde des passages avec celui des théâtres. 1822, date qu'indique W. Benjamin comme développement de ces formes ou de cet « appareil », est importante dans l'histoire des théâtres parisiens : premier éclairage au gaz à l'Opéra avec l'opéra-féérie Aladin ou la Lampe merveilleuse, dans des décors de Cicéri et Daguerre. Cette même année, Daguerre ouvre son Diorama, rue Samson (à l'emplacement de l'actuelle Place de la République, alors Place du Château d'Eau), variante de Panorama combinant la peinture à des variations de lumière ${ }^{34}$. C'est un nouvel âge perspectif qui envahit le spectaculaire et les scènes de théatre. Il n'est pas anodin de rappeler que Daguerre, l'un des inventeurs de la photographie, a eu son heure de gloire scénographique ${ }^{35}$. Si le mot appareil a été remplacé au théâtre par celui de " grand spectacle » ou de "féerie » (réapparaissant bientôt dans la photographie, la chimie, etc.), on voit bien que le concept s'est adapté à cette nouvelle visualité que Théophile Gautier nomme « le temps des spectacles purement oculaires ${ }^{36}$ ».

On voit là très matériellement comment «l'appareil perspectif », dans son évolution théâtrale (de la perspective oblique des Bibbiena et de Giovanni Niccolò Servandoni à la création d'un "milieu » et de plans de lumières et d'ombres sur la scène), est concrètement lié à la naissance de la photographie et au monde des passages. Ces 
appareils se répondent, comme c'est le cas du musée moderne, sous la forme primitive de la Galerie qui le voit naître : avatar de la galerie princière, à la française ou à l'italienne, Galerie du Louvre rêvée et ruinée par Hubert Robert et première galerie publique muséale à Dulwich à Londres, etc. Il est curieux de voir la transformation lente et profonde de ces appareils d'architecture de la Stoa Poikilè et des péristyles antiques aux formes urbaines, théâtrales, commerciales et muséales. Leur importance pour l'architecture et la scénographie théâtrales se combine avec celle de l'arc de triomphe, appareil antique par excellence et l'un des prototypes du cadre de scène moderne dérivé de la scaenae frons romaine.

Le théâtre, lieu architectural, social et urbain, est bien aussi, en ce sens benjaminien, un appareil. De la pierre à la toile, aux effets de lumière et aux tracés urbains jusqu'aux tirages argentiques, c'est une histoire de la projection visuelle qui semble proposée à l'esthétique. Si Le Livre des passages est bien fondateur d'une esthétique moderne, il faut l'inscrire dans une généalogie des formes et des pratiques qui inclut le théâtre.

Le débordement de l'appareil perspectif scénique, d'abord vers l'architecture du théâtre, puis vers l'architecture de la représentation urbaine témoigne sans aucun doute d'une démocratisation et d'une commercialisation de l'appareil visuel. À la charnière de la Révolution française, du Directoire, du Consulat et de l'Empire et finalement au cœur de la Restauration, il va de pair avec la liberté (relative) des théâtres, l'esprit d'entreprise et de conquête, un éclectisme de la mode parisienne. Alors que les œuvres d'art deviennent accessibles, visibles dans les galeries des musées, et d'abord au Muséum central des arts, avant les immenses galeries industrielles et commerciales des Expositions universelles, la marchandise se démocratise ou s'embourgeoise, comme on voudra, dans l'espace de la "capitale du xix siècle ». L'appareil déborde l'espace scénique pour envahir ces sortes de rues, dégagées des embarras de la circulation que sont les passages parisiens entre intérieur et extérieur. La scène elle-même revendique sa part de réalité bourgeoise dans le drame et de somptuosité onirique dans la féerie ou le grand opéra. C'est ainsi que l'appareil scénique se transforme en son sens illusionniste ou disparaît au $\mathrm{xx}^{\mathrm{e}}$ siècle pour redevenir constructif, tout comme la perspective s'efface de la peinture pour réapparaître dans ses éléments constitutifs avec l'avant-garde des années 1910-1920. Le sens même d'appareil scénique disparaît peu à peu. Dans un ouvrage récent consacré à l'histoire du théâtre antique ${ }^{37}$, il désigne les accessoires, les costumes, les masques et les machines, tout ce qui fait la matérialité de la scène (skeuai grecs ou res scenica latine). Si le terme s'efface, la réalité des éléments matériels demeure dans une tension constitutive entre variété et refus qui doit conduire à s'interroger sur un autre sens possible de la notion du côté de l'acteur.

\section{BIBLIOGRAPHIE}

Aristote, Poétique, trad. M. Magnien, Paris, Le Livre de poche, 1990.

Beaumarchais Pierre Caron de, Euvres, Paris, Gallimard, coll. « Bibliothèque de la Pléiade », 1988. 
Benjamin Walter, Paris, capitale du XIX ${ }^{e}$ siècle. Le livre des passages, trad. J. Lacoste, Paris, Le Cerf, 1993.

Cardinali Philippe, Marc Perelman (dir.), Ville et architecture en perspective, Nanterre, Presses universitaires de Paris Nanterre, 2018.

Comment Bernard, Le XIX siècle des Panoramas, Paris, Adam Biro, 1993.

Déotte Jean-Louis, L'homme de verre : esthétiques benjaminiennes, Paris, L'Harmattan, 1998.

Déotte Jean-Louis, L'époque de l'appareil perspectif: Brunelleschi, Machiavel, Descartes, Paris, L'Harmattan, 2001.

Diderot Denis et Jean Le Rond dit d'Alembert (dir.), Encyclopédie ou Dictionnaire raisonné des sciences, des arts et des métiers, Paris, Briasson/David/le Breton/Faulche, 1751-1765, Gallica : https://catalogue.bnf.fr/ark :/12148/cb35153871q

Diderot Denis, CEuvres, t. IV, Paris, Robert Laffont, 1996.

Diderot Denis, Le Rêve de d'Alembert, Paris, GF-Flammarion, 2002.

Fontenelle Bernard Le Bouyer de, Entretiens sur la pluralité des mondes, Paris, Verviers, coll. « marabout université », 1973.

Font-Réaulx Dominique de, «Le vrai sous le fantastique. Esquisse des liens entre le daguerréotype et le théâtre de son temps » in Études photographiques, nº 16, mai 2005.

Font-Réaulx Dominique de, «Les origines théâtrales de la photographie » in Actes des congrès de la Société française Shakespeare [En ligne], 33 | 2015, URL : http://journals.openedition.org/ shakespeare/3052; DOI : 10.4000/shakespeare.3052

Frantz Pierre, L'esthétique du tableau dans le théâtre du XVIII siècle, Paris, PUF, 1998.

Frantz Pierre, Wynn Thomas (dir.), La scène, la salle et la coulisse dans le théâtre du XVIII siècle en France, Paris, PUPS, 2011.

Furetière Antoine, Dictionnaire universel..., La Haye, 1690. Gallica : https://gallica.bnf.fr/ark :/ 12148/bpt6k50614b/f111.item

Gonzaga Pietro di, La Musique pour les yeux et l'optique théâtrale - opuscules tirés d'un plus grand ouvrage sur le sens commun..., Saint-Pétersbourg, Imprimerie Nationale, 1800 [seconde édition 1807].

Guillot Catherine, « Richelieu et le théâtre », Transversalités, vol. 117, no 1, 2011, p. 85-102. https://doi.org/10.3917/trans.117.0085

Jomaron Jacqueline de (dir.), Le Théâtre en France, 2 vol., Paris, Armand Colin, 1988-1989.

Konigson Élie, Le Théâtre dans la ville, Paris, CNRS Éditions, 1988.

Le Guen Brigitte, Milanezi Silvia (dir.), L'appareil scénique dans les spectacles de l'Antiquité, Saint-Denis, Presses universitaires de Vincennes, 2013.

Ledoux Claude Nicolas, L'architecture considérée sous le rapport des arts, des mœurs et de la législation, Paris, Hermann, 1997.

Martin Roxane, L'Émergence de la notion de mise en scène dans le paysage théâtral français (1789-1914), Paris, Classiques Garnier, 2013.

Mottola Molfino Alessandra, Il libro dei musei, Torino, Allemandi, 1992.

Pasquier Pierre (dir.), Le Mémoire de Mahelot, Paris, Champion, 2005. 
Pougin Arthur, Dictionnaire historique et pittoresque du théâtre et des arts qui s'y rattachent..., Paris, Firmin-Didot, 1885, consultable sur https://gallica.bnf.fr/ark :/12148/bpt6k2043674.

Sabbattini Nicola, Pratique pour fabriquer scènes et machines de théâtre, trad. M. et R. Canavaggia et L. Jouvet, Neuchâtel, Ides et Calendes, 1994.

Serlio Sebastiano, Il primo libro d'architettura... Il secondo libro di perspettiva... Le premier livre d'architecture... Le second livre de perspective..., mis en langue francoyse, par Jehan Martin..., Paris, Jehan Barbé, 1545. Bibliothèque numérique INHA : https://bibliothequenumerique.inha.fr/ viewer $/ 12652 /$ ?offset =\#page =138\&viewer $=$ picture \&o =bookmark\&n $=0 \& q$

Surgers Anne (éd.), La Pellegrina et les Intermèdes. Florence, 1589, Vijon, Lampsaque, 2010.

Voltaire, La tragédie de Sémiramis, Paris, Le Mercier et Lambert, 1749, consultable sur Gallica : https://gallica.bnf.fr/ark :/12148/bpt6k5783896g/f24.image.

Prinz Wolfram, Die Entstehung der Galerie in Frankreich und Italien, Berlin, Mann, 1970. Traduction italienne : Galleria. Storia e tipologia di uno spazio architettonico, a cura di C. Cieri Via, Modena, Panini, 2006.

\section{NOTES}

1. Voir le vers célèbre de Racine dans Britannicus (acte II, scène II) : «Belle, sans ornement, dans le simple appareil/D'une beauté qu'on vient d'arracher au sommeil. »

2. Furetière, Dictionnaire universel..., La Haye, A. et R. Leers, 1690. Gallica : https://gallica.bnf.fr/ ark :/12148/bpt6k50614b/f111.item

3. TLFi : http://atilf.atilf.fr/tlf.htm s. v. Appareil.

4. Les vols sont les effets de machinerie, comportant souvent des chars, des apparitions dans le ciel des opéras et spectacles baroques. Ils remontent à l'ingénierie théâtrale italienne du $\mathrm{XVI}^{\mathrm{e}}$ siècle (notamment Buontalenti).

5. Encyclopédie ou Dictionnaire raisonné des sciences, des arts et des métiers, vol. I, 1751, p. 543. s. v. Appareil.

6. Voir l'essai florentin d'Aby Warburg, in: La Pellegrina et les Intermèdes. Florence, 1589, présentation Anne Surgers, Vijon, Lampsaque, 2010, suivi d'une « Description de l'appareil et des Intermèdes faits pour la comédie représentée à Florence... ", édition originale, 1589: https:// gallica.bnf.fr/ark :/12148/bpt6k135085x.image.

7. Le Second livre de perspective de Sebastian Serlio Bolognais mis en langue française par Jehan Martin, Paris 1545, p. 65 . Orthographe modernisée, c'est nous qui soulignons. Bibliothèque numérique INHA : $\quad$ https://bibliothequenumerique.inha.fr/viewer/12652/ ? offset $=\#$ page $=138 \&$ viewer $=$ picture \&o $=$ bookmark\&n $=0 \& q$

8. Ibid., p. 66. Jean Martin traduit cette fois par « équipages des comédies ».

9. Ces premiers succès de Torelli avaient été précédés par l'intervention de Giovan Maria Mariani pour l'inauguration de la grande salle du Palais-Cardinal (Palais-Royal) qui abritera plus tard le théâtre de Molière et, après la mort de ce dernier, l'Opéra de Lully, voir l'Introduction de Pierre Pasquier à sa réédition du Mémoire de Mahelot, Paris, Champion, 2005, p. 46 et p.185-187 et Catherine GUILLOT, « Richelieu et le théâtre », Transversalités, vol. 117, n 1, 2011, p. 85-102.

10. Russell Goulbourne « Espace théâtre, espace politique : La Princesse de Navarre de Voltaire » in Frantz Pierre et Thomas Wynn (dir.), La scène, la salle et la coulisse dans le théâtre du XVIII siècle en France, Paris, PUPS, 2011, p. 225-234 et l'article «Fêtes à la cour de France » rédigé par Cahusac dans l'Encyclopédie. 
11. La tragédie de Sémiramis par M. de Voltaire, Paris, Le Mercier, 1749, p. 23 consulté sur Gallica: https://gallica.bnf.fr/ark :/12148/bpt6k5783896g/f24.image. C'est nous qui soulignons.

12. Aristote, Poétique, chap. VI, 1450 b, trad. M. Magnien, Paris, Le Livre de poche, 1990. Charles É mile Ruelle en 1883 traduit opsis par « appareil scénique ».

13. L'énumération de ses articles forme un curieux inventaire: Culte, Dévouement, Druide, Entrées, Évocation des mânes, Excommunication, Feu de joie, Isis, Juge des enfers, Junon, Mitre, Mois des Égyptiens, Rois de Rome, Spectacles, Phantome, Jeux olympiques, Poésie épique, Proserpine, Religion des Grecs et des Romains, Jeux séculaires, Théâtre des Grecs, Triomphe, Vêtements des Chrétiens...

14. Articles Déserteur, Ecclésiastique, Effigie, Fanfare, Funérailles, Juridiction économique, Justice, Mort, Peine, Prague.

15. Encyclopédie ou Dictionnaire raisonné des sciences, op. cit., vol. XIII, 1765, p. 12.

16. Ibid., vol. I, 1751, p. 543.

17. Article Genève.

18. Article Gloire, Glorieux, Glorieusement, Glorifier.

19. Article Méthode.

20. Voir Michèle Sajous D’Oria, «Une scène pour Sémiramis » in Frantz Pierre et Thomas Wynn (dir.), La scène, la salle et la coulisse..., op. cit., p. 125-149 et Pierre Frantz, L'esthétique du tableau dans le théâtre du XVIII siècle, Paris, Puf, 1998, p. 45 et suiv.

21. Beaumarchais, Euvres, Paris, Gallimard, coll. «Bibliothèque de la Pléiade », 1988, p. 133. C'est nous qui soulignons.

22. Id.

23. Arthur Pougin, Dictionnaire historique et pittoresque du théâtre et des arts qui s'y rattachent..., Paris, Firmin-Didot, 1885, consulté sur https://gallica.bnf.fr/ark:/12148/bpt6k2043674, p. 46. C'est nous qui soulignons.

24. Aristote, Poétique, 49a18, op. cit.

25. «Les passages sont des maisons ou des corridors qui n'ont pas de côté extérieur - comme le rêve ", Walter Benjamin, Paris, capitale du XIX siècle. Le livre des passages, Paris, Le Cerf, 1993, p. 424 ou « La superstructure est l'expression de l'infrastructure. [...] Le collectif exprime tout d'abord ses conditions de vie. Celles-ci trouvent leur expression dans le rêve et leur interprétation dans le réveil ", Ibid. p. 410.

26. Voir plusieurs articles dont ceux de Jean-Louis Déotte, Christian Biet et le nôtre in Philippe Cardinali et Marc Perelman (dir.), Ville et architecture en perspective, Nanterre, Presses universitaires de Paris Nanterre, 2018.

27. L'expression appartient au scénographe russe, actif à la cour de Russie, Pietro di Gottardo Gonzaga (1751-1831) et forme le titre de son traité La musique des yeux et l'optique théâtrale..., SaintPétersbourg, 1800.

28. Fontenelle, Entretiens sur la pluralité des mondes, Paris, Verviers, coll. marabout université, 1973, p. 29-30.

29. Diderot, Paradoxe sur le comédien in Euvres, t. IV, Paris, Robert Laffont, 1996, p. 1383.

30. Ibid., p. 1382-1383.

31. Élie Konigson, « Autour d'un théâtre : le quartier Graslin à Nantes », in Le Théâtre dans la ville, Paris, CNRS Éditions, 1988.

32. Voir Wolfram Prinz, Die Entstehung der Galerie in Frankreich und Italien, Berlin, Mann, 1970, consulté dans sa traduction italienne: Wolfram Prinz, Galleria. Storia e tipologia di uno spazio architettonico, Modena, Panini, 2006.

33. Walter Benjamin, Paris, capitale du XIXe siècle..., op. cit., p. 880.

34. Bernard Comment, Le XIX ${ }^{e}$ siècle des Panoramas, Paris, Adam Biro, 1993.

35. Sur tout ceci, voir Roxane Martin, L'Émergence de la notion de mise en scène dans le paysage théâtral français (1789-1914), Paris, Classiques Garnier, 2013, p. 85-94 et illustration ainsi que 
Dominique de Font-Réaulx, «Les origines théâtrales de la photographie », Actes des congrès de la Société française Shakespeare [En ligne], 33 | 2015, URL: http://journals.openedition.org/ shakespeare/3052; DOI : 10.4000/shakespeare.3052, article paru antérieurement avec des illustrations similaires sous le titre «Le vrai sous le fantastique. Esquisse des liens entre le daguerréotype et le théâtre de son temps », Études photographiques, n 16, mai 2005.

36. Olivier Bara, «Avant-propos ", Orages $\mathrm{N}^{\circ} 4$, Boulevard du crime : le temps des spectacles oculaires, mars 2005, p. 9-20. http://orages.eu/wp-content/uploads/2012/12/Or4_p9.pdf

37. Brigitte Le Guen et Silvia Milanezi (dir.), L'appareil scénique dans les spectacles de l'Antiquité, Saint-Denis, Presses universitaires de Vincennes, 2013.

\section{AUTEUR}

\section{STÉPHANE POLIAKOV}

Université Paris 8 (EA 1573). Il est également coordonnateur du thème Créations, Pratiques,

Public de l'axe $1:$ Industries de la culture et arts à la MSH Paris Nord 